\title{
Die zweite Tafel der Serie Kagal in Hattusa
}

\author{
von G. Wilhelm - Würzburg
}

Im 62. Band dieser Zeitschrift hat E. von Weiher das 1933 in Boğazköy, genauer in dem Gebäude A, Raum 6 Nord auf Büyükkale, gefundene Fragment eines sumerisch-akkadischen Vokabulars $(2545 / \mathrm{c}=\mathrm{KBo} .16,87)$ bearbeitet $^{\prime}$. Er konnte für den Text, der zuvor von E. Laroche als CTH 309 unter den ,fragments non identifiés" aufgeführt worden war, Parallelen in Izi Boğazköy A, Kagal Tafel I und den grammatikalischen Listen OBGT Ia, II und NBGT III nachweisen und dadurch die größtenteils fehlende sumerisch-logographische Spalte und die ersten neun weitgehend abgebrochenen akkadischen Äquivalente ergänzen.

CAD N/1, 292a, zitiert unter napharu die Zeilen Vs. ii 10'-11', ohne den Text mit einer der bekannten Serien in Verbindung zu bringen, doch klassifiziert CAD N/2, 56b, das Zitat von Rs. v 15'$18^{\prime}$ mit dem Zusatz "(Kagal)“, und ebenso S. 208b unter nidnu die Zeile Rs. v 20'.

Die Arbeit an den noch unveröffentlichten Fragmenten (sume-

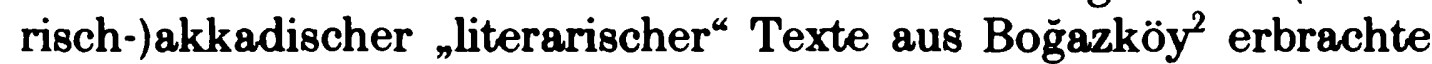
einen Zusammenschluß von $2545 / \mathrm{c}$ mit dem nur einseitig erhaltenen unveröffentlichten Fragment 1970/c (KBo. 36,1), das ebenfalls in Gebäude A, und zwar im Tafelschutt des nördlichen Teils von Raum 5, gefunden wurde. Durch das Anschlußfragment werden die durch von Weiher ergänzten akkadischen Äquivalente

1 E. von Weiher, ZA 62 (1972) 109-114.

2 Für die Úberlassung dieses Materials zur Edition und Bearbeitung bin ich H. Otten ebenso zu Dank verpflichtet wie für die Genehmigung, die Sammlungen der Arbeitsstelle Boğazköy-Forschung der Akademie der Wissenschaften und der Literatur zu Mainz benutzen zu dürfen. Der genannten Akademie danke ich herzlichst für eine Reisebeihilfe, die es mit unter anderem erlaubte, die Originalfragmente im Anadolu Medeniyetleri Müzesi zu Ankara einzusehen und die Textzusammenschlüsse auszuführen. Der Leitung dieses Museums sowie den Kuratorinnen der Tontafelabteilung, Frau Gül Ün und Frau Ayß̧e Karaduman, schulde ich Dank für die Gewährung angenehmer Arbeitsmöglichkeiten und stete Hilfsbereitschaft. 
in den Zeilen Vs. ii $1^{\prime}-1 l^{\prime}$ gewonnen. Gegenüber den Ergänzungen ergeben sich unerwartete bedeutsame Abweichungen. Weiterhin beantwortet das Anschlußfragment von Weihers Frage, „ob sich daran noch eine weitere Spalte mit der hethitischen Übersetzung anschließt ${ }^{\alpha 3}$, negativ. Nach einem Kolumnentrenner folgt vielmehr eine Kolumne von sumerischen Wörtern, deren Logogramm mit dem Zeichen A beginnt.

Durch den weiteren Anschluß des als KUB 30,5 publizierten, ebenfalls im nördlichen Teil des Raumes 6 in Gebäude A gefundenen Fragments 1719/c (sic!, 2488/c ist KUB 30,4!) ergibt sich, daß auch diese Kolumne ebenso wie die linke durch eine senkrechte Linie unterteilt war, die die sumerischen Wörter von ihren Ausspracheangaben und akkadischen Äquivalenten trennte. KUB 30,5 wurde bisher als einziger Textzeuge der altbab. grammatikalischen Texte (OBGT) in Boğazköy betrachtet. ${ }^{4}$

Der Text stellt sich nun folgendermaßen dar:

Vs. ii

$$
\begin{aligned}
& 1^{\prime} \text { [ } \\
& 2^{\prime} \text { [gú-ri-a] } \\
& 3^{\prime} \text { [gú-e-a] } \\
& \left.4^{\prime}[g u ́-s ̌ e]-\Gamma_{a}\right] \\
& 5^{\prime}[\mathrm{gú}-\mathrm{re}]-\mathrm{e} \\
& 6^{\prime}[\mathrm{gú}]-\Gamma_{\mathrm{e}} \mathrm{l}^{\mathrm{l}} \mathrm{e} \\
& 7^{\prime}[\mathrm{gú}-\mathrm{z} e]-\mathrm{e} \\
& 8^{\prime}[\mathbf{g u ́}-\mathbf{r}] \mathbf{i}-\mathbf{t a} \\
& 9^{\prime}[\mathrm{gú}-\mathrm{e}-\mathrm{t}] \mathrm{a} \\
& 10^{\prime}[g u ́-\gamma e-t] a \\
& \text { 11' [gú-si] } \\
& 12^{\prime} \text { [gú-si-kur-r]a } \\
& 13^{\prime} \text { [gú-gúru] } \\
& \text { 14' [gú-gúru] } \\
& \text { 15' [ gú-gigirum] } \\
& 16^{\prime} \text { [ér] } \\
& 17^{\prime} \text { [ér] } \\
& \text { 18' [ér] }
\end{aligned}
$$

]

${ }^{3}$ von Weiher, l.c. 109.

4 MSL 4, 128; E. Laroche, CTH Nr. 307.

$\left[\mathrm{gu}_{5}\right.$-u-ri-i]a [ $\left.\left.\backslash a\right] n-n i-\Gamma_{k e-e}\right]$ $\left[\mathrm{gu}_{5}-\mathrm{u}\right] \cdot \Gamma_{\mathrm{e}} \mathrm{T}_{-\mathrm{ia}}[]$an-ni-ke-e $\mathrm{gu}_{5}-\mathrm{u}-[\mathrm{r}] \mathrm{e}-\mathrm{ia}[\backslash]^{\top} \boldsymbol{C}^{\top}-n u-m i-k e-e$ $\mathrm{gu}_{5}$-u-re-es $\backslash[u] l-l i-i \xi$ $\mathrm{gu}_{5}$-u-e-es $\backslash \Upsilon_{a n}{ }^{\top}-n i-i \S$ $\mathrm{gu}_{5}$-u-si-is $\backslash a-n u-{ }^{\top} u^{\top}-m i-i \xi$ $\mathrm{gu}_{5}$-u-ri-it-ta $\left\lceil i^{\prime}\right\}^{\prime}-\mathrm{tu} u l-l i-k a m$ $\mathrm{gu}_{5}$-u-e-et-ta $i \xi$-tu $a[n-n] e^{-}-e$ $\mathrm{gu}_{5}$-u-se-et-ta is-tu $a-\Gamma_{n u-m i}$ ke-e $\mathrm{gu}_{5}-\mathrm{u}-\mathrm{si}_{17}$ na-ap-ha-ru $\mathrm{gu}_{5}-\mathrm{u}-\mathrm{si}_{17}-\mathrm{ku}-\mathrm{u}-\mathrm{ra}$ na-ap-〈ha- $\rangle a r$ $[m a]-{ }^{\top} a^{\top}-t i$ $\mathrm{gu}_{5}-\mathrm{u}-\mathrm{gu} \mathrm{u}_{5}-\mathrm{ru} k a-n a-a-s^{2} u$ $g u_{5}-\mathrm{u}-g u_{5}-\mathrm{ru} k a-m a-a-{ }^{\top} s u_{\mathrm{x}}{ }^{\top}(\mathrm{SU})$ $\mathrm{gu}_{5}$-u-ga-ag-re $k i-t a-m u-s u_{\mathrm{x}}(\mathrm{SU})$ ar $d i_{12}-i m-t u ̀$ ar bi-ki-it-tì ar ta-az-zi-im-tù 


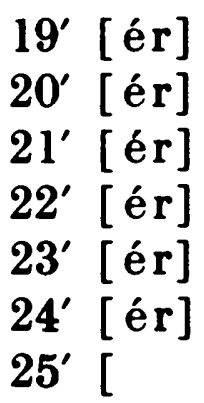

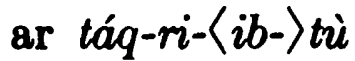

ar ta-às-li-tù

ar ta-a-ni-bu

ar $g i_{5}-r a-a-n u$

ar $e n_{6}-n e ́-e[t]-t[u]$

$\left.r_{\mathbf{a r}}\right]^{\mathrm{x}}[$

]x[

V8. iii $1^{\prime}$ a $\times \times$ [

$2^{\prime}$ a-pú-pú [

$3^{\prime}$ a-pú-8ag [

$4^{\prime} a-d u ̀ g-[g a$

$5^{\prime}$ id-da [

6' (rad.)

$7^{\prime} \mathbf{a}-\mathbf{a} \mathbf{b}-\mathbf{b}[\mathbf{a}$

$8^{\prime}$ a-làl [

$9^{\prime} \quad \mathrm{a}-\mathrm{zi}-\mathrm{g}[\mathrm{a}$

$10^{\prime} \mathrm{a}-\mathrm{zag} \cdot \mathrm{ba} \mathrm{r}$ ?

$11^{\prime} \mathrm{a}-[\mathrm{s}] \mathrm{ur}-[\mathrm{r}] \mathrm{a}$

$\left.12^{\prime} \Upsilon_{\mathrm{a}} \Upsilon_{-8 \mathrm{u}} \mathrm{r}\right] \cdot \mathrm{ra}$ [

$13^{\prime} \Upsilon^{\prime}{ }^{\top}-[b] i$ ?-na

$14^{\prime} \mathrm{a}-[\mathrm{b}] \mathrm{i}$ ?-n a

$\left.15^{\prime} \Upsilon_{\mathbf{a}}\right]^{\prime}[$

Rs. iv $1^{\prime} \mathrm{x}[$

$2^{\prime} \mathbf{a}-[\mathbf{x}] \mathbf{x}$ x $[$ ]

$\left.\mathbf{3}^{\prime} \boldsymbol{r}_{\mathbf{a}}\right\rceil_{-[}[\mathrm{b}] \mathbf{a}$

$4^{\prime}[a-b a]-k a m$

$5^{\prime}\left[a-b a-g_{7}-n\right] a m$

$6^{\prime}\left[a-b a-g i n_{7}-n a m\right.$ a $] l-d u$

i-ne!?-gii al-du ki-ma $m a-a n-n i \times \times \times \times$

a-bé-a ma-an-nu

a-bé-a-ka ma-an- $n a^{\top}-\delta^{\circ} u$

a-bá-a-gi ${ }_{5}$-nam ki-ma ma-an-[ni]

a-bá-a-gi ${ }_{5}$-nam al-du

ki-ma $m[a-a n-n i]$

$\backslash$ ta-al-la-ak

Für Rs. v vergleiche man die Bearbeitung von Weihers.

Die im Text verwendeten Lautwerte zeigen die für das Syllabar der Randgebiete typische Sonoritätsindifferenz: PA = bá Rs. iv 3'$6^{\prime}$; TA = dá Rs. v 19sq.; TE $=d i_{12}$ Vs. ii 16'; DU = tù Vs. ii 16'-20', $23^{\prime} ; \mathrm{KI}=g e_{5}, g i_{5}$ V8. ii $22^{\prime}$, Rs. v 3', 9', 12'; KU = gu $u_{5}$ Vs. ii passim. Außerdem werden 8 -haltige Zeichen zur Darstellung von /s/ verwendet: $\mathrm{AS}=a ̀ s$ Vs. ii $20^{\prime}$; $\mathrm{SI}_{\mathrm{I}}=s i_{17}$ Vs. ii 11'sq.; SU $=s u_{x}$ Vs. ii $14^{\prime}$ sq. 
Die paläographische Untersuchung der Liste fuihrt zu dem Ergebnis, daß von den Zeichenvarianten, die in hethitischen Texten aus Boğazköy besonders zuverlässig als Alterskriterium verwendet werden können, durchgehend die älteren Formen erscheinen: AG Vs. ii 15'; IG Rs. v 19'(?); NI Vs. ii $3^{\prime}, 6^{\prime}, 9^{\prime}, 21^{\prime}, 23^{\prime}$,

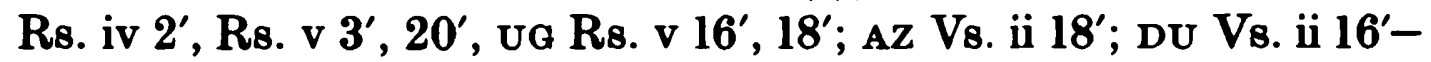
$20^{\prime}, 2^{\prime}$, Rs. v $12^{\prime}, 16^{\prime}, 18^{\prime}$; AL Rs. iv $2^{\prime}, 6^{\prime}$; LI Vs. ii $5^{\prime}, 8^{\prime}, 20^{\prime}$, Rs. v $10^{\prime}, 13^{\prime}, 19^{\prime}$.

Der Text dürfte demnach von einem Schreiber der HattusaSchule vor dem 13. Jh. geschrieben worden sein.

Die akkadischen Wörter, die das bisher unveröffentlichte Fragment 1970/c bietet, weichen teilweise von den Gleichungen ab, die die Paralleltexte liefern. Wie schon von Weiher gesehen hat,

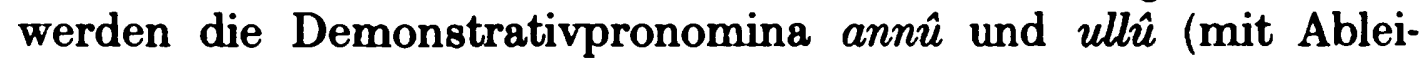
tungen) gegenüber den Paralleltexten vertauscht. Dieser Fehler findet nun seine Erklärung darin, daß der Schreiber in Vs. ii 3' versehentlich das an-ni-ke-e von Zeile $2^{\prime}$ wiederholte und dadurch die Reihenfolge in den Dreiergruppen der Demonstrativa im folgenden verwechselte.

Besonders auffällig ist es, daß unser Text $-k \imath^{3} a m$ mit der aus Mari bekannten Kontraktion ia $>e$ aufweist. Der parallele Abschnitt in Izi Boğazköy A 170 sqq. bietet - anders als die kanonische Serie ká-gal = abullu Tafel I 377-379 mit annis, ullis, anaummis - ebenfalls Formen, die auf $-k i$ am zurückgehen, jedoch in der mittelbab. zu erwartenden Gestalt $-k \hat{a}([u l l i k] \hat{a},[\ldots] k \hat{a})$. Dies gibt einen Hinweis auf den Traditionsweg unserer Liste, der durch ein westsemitisch geprägtes Milieu der altbab. Zeit verlaufen sein muß. - Für das akkadische Wörterbuch wird aus VB. ii 4' und 10 ' der Eintrag anummikê "dort" gewonnen.

In der altbab. Liste Proto-Kagal folgen auf den Komplex „Tore und Bauwerke ${ }^{*}$, der die ersten 233 Zeilen einnimmt, sogleich die mit dem Zeichen A beginnenden Wörter ${ }^{5}$. Ein Abschnitt von mit gú anfangenden Wörtern, der in unserem Fragment dem AAbschnitt vorausgeht, gehört im älteren altbab. Kanon von Nippur zu der Liste Nigga ${ }^{6}$. Diese Liste wurde als selbständige Komposition anscheinend nicht weiter tradiert, das in ihr verarbeitete Material wurde vielmehr in andere akrographische Listen einge-

\footnotetext{
5 MSL 13,63.

${ }^{6}$ Nigga 419-489; cf. MSL 13,107 sqq.
} 


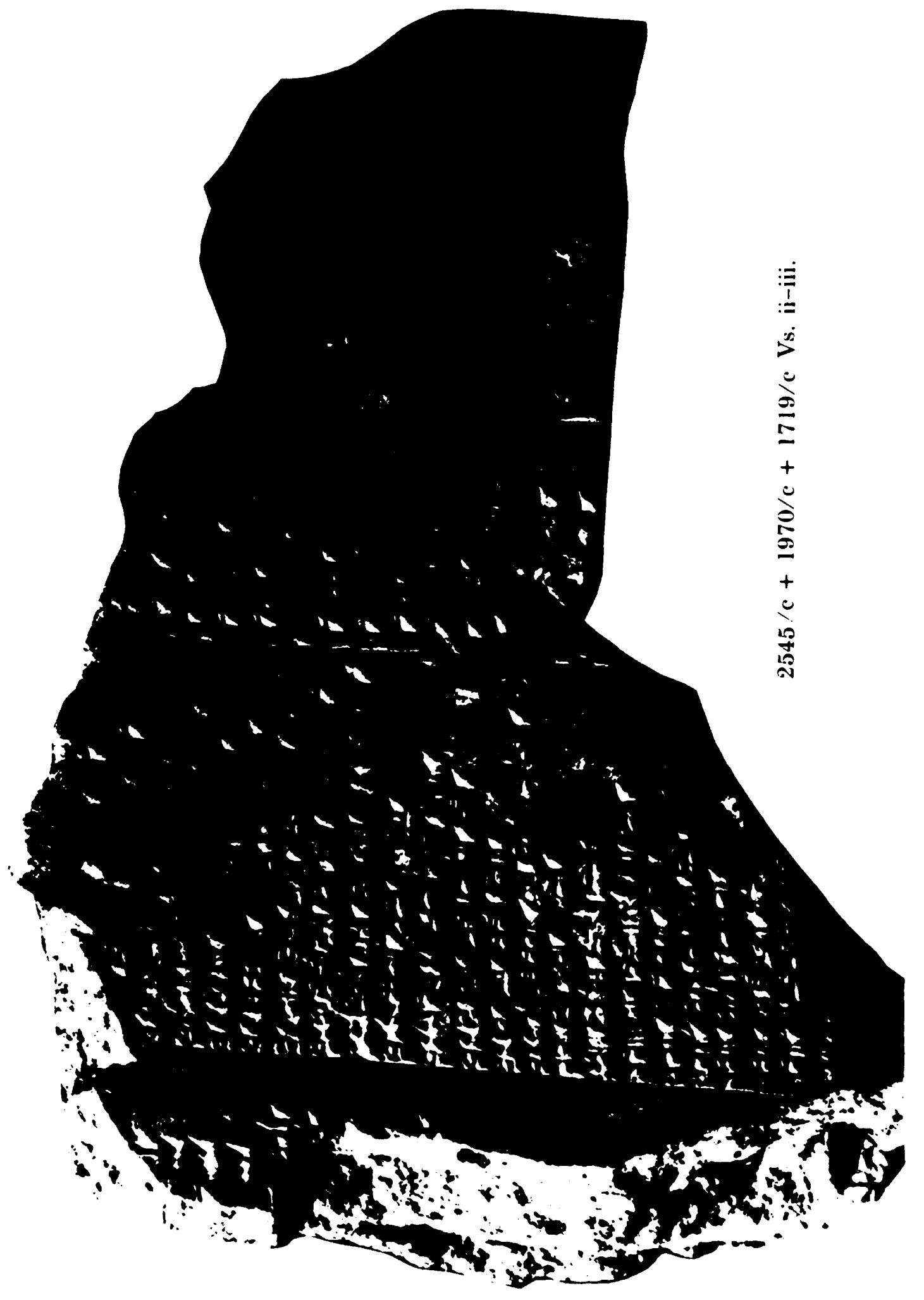




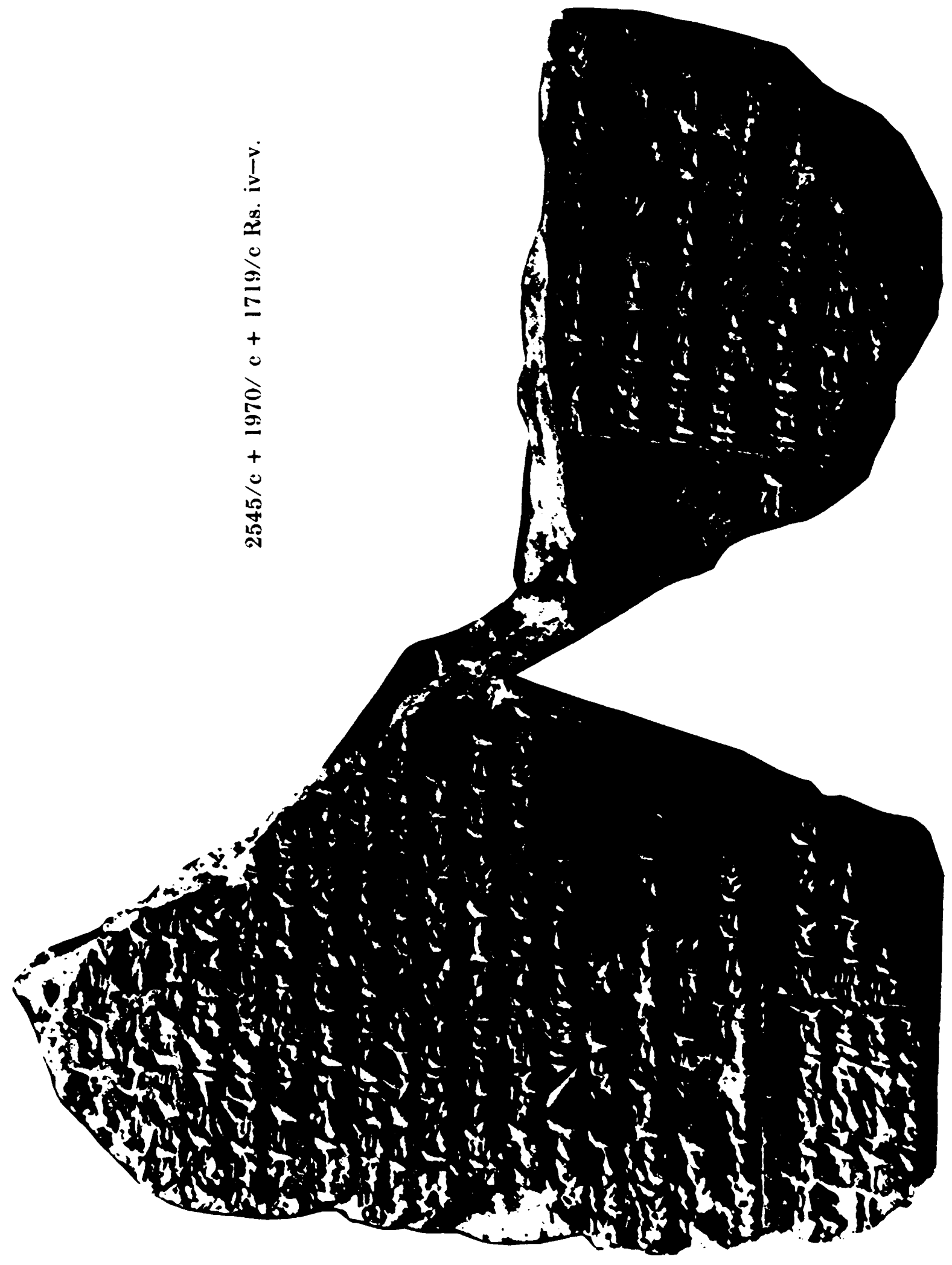


fügt ${ }^{7}$. Daher findet sich der gú-Abschnitt bereits in einer spätaltbab. Fassung von Kagal ${ }^{8}$. Er steht dort am Ende der I. Tafel nach mehreren Abschnitten, die gleichfalls ursprünglich nicht zu Kagal gehörten ${ }^{9}$. Ein Teil der gú-Einträge stammt aber aus den altbabylonischen grammatikalischen Texten ${ }^{10}$.

In der "kanonischen" Serie ká-gal = abullu ist der sehr lange AAbschnitt, der in Proto-Kagal zwischen den mit é und den mit gi i beginnenden Einträgen steht, ganz ausgelassen oder doch zumindest stark gekürzt worden ${ }^{11}$. Eine durch ihren Kolophon als Teil der Serie $\mathrm{ká}-\mathrm{gal}=$ abullu ausgewiesene Tafel der Bibliothek Tiglatpilesers I. umfaßt, soweit erhalten, ausschließlich mit A beginnende Wörter ${ }^{12}$. Der A-Abschnitt ist also wohl wegen seiner großen Länge zumindest teilweise (s. unten) aus seinem ursprünglichen Zusammenhang herausgelöst und an anderer Stelle des Serienwerks eingefügt worden.

Aus Hattusa ist die Serie Kagal bisher nur mit ihrer ersten Tafel bekannt ${ }^{13}$. Es sind uns mindestens zwei Exemplare dieser Tafel teilweise erhalten, von denen eines, KBo. 26,40 (dazu o.A. KBo. 1,59?), aus dem Bereich des Großen Tempels stammt, während ein anderes (Bo. 5067(t)? Bo. 1520(t)? Bo. 46(t)? 1749/c(t)? $605 / b)^{14}$ - falls die Fragmente wirklich zur gleichen Tafel gehören - auf Büyükkale aufbewahrt wurde. Die Tafel I der Serie Kagal Boğazköy umfaßte, nach den erhaltenen Fragmenten zu schließen, die Abschnitte ká-gal, kisal, ká und é. Da von der Rückseite der Tafel nur Teile der an den rechten Rand grenzenden Kolumne erhalten sind, könnten weitere Abschnitte folgen, nämlich die in der kanonischen Fassung in Kolumne $v$ gebuchten Einträge zu g is und vielleicht auch noch die dort in Kolumne vi aufgeführten Einträge zu gìr und dùg.

Das hier vorgelegte Textstück $2545 / c+1970 / c+1719 / c$ kann nicht zu dieser Tafel gehören, da es in den an den rechten Rand

Cf. M. Civil, MSL 13, 92: 125.

"Canonical ká·gal = abullu" Tafel I; cf. MSL 13, 227.

Cf. MSL 13, $230 \mathrm{sq}$.

OBGT Ia Kol. 2'-7'; cf. MSL 4, 63.

Cf. M. Civil, MSL 13, 227.

Cf. MSL 13, 232 sq.

MSL 13, 148-153.

16 KUB 30,8 (t)? KUB 3,102(+)? KBo. $2,28(+)$ ? KUB 30,6 (t)? KUB 30,7; zur möglichen Zusammengehörigkeit cf. MSL 13, 148 mit Hinweis auf die Bemerkungen der Herausgeber sowie die Notizen Güterbocks. 
reichenden Kolumnen auf Vorder- und Rückseite Wörter des AAbschnittes bucht. Die Bestimmung von Vs. und Rs. der Fragmente 2545/c und $1719 / \mathrm{c}$, die von Ehelolf und Otten noch in Unkenntnis des Textzusammenschlusses unabhängig voneinander übereinstimmend getroffen wurde, läßt den A-Abschnitt auf den g ú-Abschnitt folgen, wie dies auch in der kanonischen Fassung der Fall ist. Nach der Angabe Ottens in der Edition ist mit der letzten Zeile der Rs. der "Rand nahe“. Der gú-Abschnitt der Vs. ist demnach im oberen Teil der Tafel zu plazieren. Da die erhaltenen Teile die letzten des gú-Abschnittes sind, dürfte dieser Abschnitt bereits in der ganz abgebrochenen Kolumne $\mathrm{i}$ beginnen, aber nicht bis an den Tafelanfang zurückführen. Vielmehr wäre hier noch ein Teil des Materials der nicht erhaltenen unteren Hälfte der Kolumne vi der kanonischen Fassung zu erwarten.

Es ergibt sich daraus, daß unser neugewonnenes Textstuick zu einer Tafel gehört, die ungefähr dort einsetzt, wo Kagal Boğazköy Tafel I aufhört, nämlich im Bereich der Kolumne vi der kanonischen Fassung (gìr, dùg, [ . . ]). Die äußeren Merkmale der Textanordnung stellen diese Tafel neben das Exemplar von Kagal I, das gleichfalls auf Büyükkale gefunden wurde: Ebenso wie dieses ist sie auf Vorder- und Rückseite mit einfachen Kolumnentrennern in je drei Kolumnen eingeteilt, die wiederum in eine schmalere linke und eine breitere rechte Spalte unterteilt sind. In der linken Spalte stehen dabei jeweils die sumerischen Wörter in "orthographischer" Schreibung, in der rechten dieselben Wörter in syllabischer Schreibung und, oft von einem Winkelhaken getrennt, die akkadischen Äquivalente. Der Schluß erscheint demnach zwingend, daß das neugewonnene Textstück die II. Tafel der Serie Kagal Boğazköy repräsentiert und die auf Büyükkale gefundene I. Tafel dieser Serie fortsetzt.

Für die kanonische Serie ká - gal = abullu ergibt sich aus diesem Befund folgendes: Die "Tafel $A^{4}$ der Assur-Fassung ${ }^{15}$ folgt unmittelbar auf die I. Tafel der Serie, wie sie vor allem mit einem Exemplar aus Nippur (Ni. 633) bezeugt ist ${ }^{16}$. Die Vermutung von M. Civil, der "Tafel A“, die mit ér beginnt, gehe eine andere Tafel mit A-Einträgen voraus, da der A-Abschnitt wahrscheinlich wie in Proto-Kagal mit $\mathrm{a}=m \hat{u}$ beginne, bestätigt sich nicht: In unserem Text folgen die ér-Einträge unmittelbar auf den gú -Abschnitt. Der

15 MSL 13, 231 sqq.

16 MSL 13, 228 8qq. 
A-A Abschnitt der Boğazköy- und damit wohl auch der („kanonischen ${ }^{\text {) }}$ Assur-Fassung ist also anders aufgebaut als in ProtoKagal, wo den mit ér beginnenden Einträgen 128 Einträge zu A unmittelbar vorausgehen. Man darf vermuten, daß dieses Material seine traditionelle Position zwischen é und gi r behielt. In dem eimzigen größeren Fragment der I. Tafel der kanonischen Fassung, $\mathrm{Ni} 633$, wäre in den völlig zerstörten Kolumnen iii und iv Platz datür vorhanden.

Ein weiterer Gewinn für die Rekonstruktion der sumerischakkadischen Schultradition in Hattusa ergibt sich aus der neuen Zuюrdnung von KUB 30,5. Die altbabylonischen grammatikalischen Texte (OBGT) sind in Hattusa als selbständige Einheit (CTH 307) nicht bezeugt. 